\begin{tabular}{|c|l|}
\hline Title & Pedal foliations and Gauss maps of hy persurfaces in Euclidean space \\
\hline Author(s) & Izumiya, Shyuichi; Takahashi, Masatomo \\
\hline Citation & Hokkaido University Preprint Series in Mathematics, 1001, 1-16 \\
\hline Issue Date & 2012-3-10 \\
\hline DOI & 10.14943/84147 \\
\hline Doc URL & http://hdl.handle.net/2115/69806 \\
\hline Type & bulletin (article) \\
\hline File Information & pre1001.pdf \\
\hline
\end{tabular}

Instructions for use 


\title{
Pedal foliations and Gauss maps of hypersurfaces in Euclidean space
}

\author{
Shyuichi Izumiya* and Masatomo Takahashi ${ }^{\dagger}$
}

March 1, 2012

\begin{abstract}
The singular point of the Gauss map of a hypersurface in Euclidean space is the parabolic point where the Gauss-Kronecker curvature vanishes. It is well-known that the contact of a hypersurface with the tangent hyperplane at a parabolic point is degenerate. The parabolic point has been investigated in the previous research by applying the theory of Lagrangian or Legendrian singularities. In this paper we give a new interpretation of the singularity of the Gauss map from the view point of the theory of wave front propagations.
\end{abstract}

\section{Introduction}

The singular point of the Gauss map of a hypersurface in Euclidean space is the parabolic point of the hypersurface where the Gauss-Kronecker curvature vanishes [1, 12]. There have been many researches on singularities of Gauss maps $[2,3,17,19]$. The pedal of the hypersurface (cf. $[6,12]$ ) is the wavefront set whose singular points are the same as the parabolic points of the hypersurface. Actually, we can show that the pedal is defined in $S^{n-1} \times \mathbb{R}$. We call it a cylindrical pedal (or, dual hypersurface) of the hypersurface [5, 12, 20]. By definition, the Gauss map is the $S^{n-1}$-component of the cylindrical pedal. In this paper we consider the $\mathbb{R}$ component of the cylindrical pedal which defines a function on the hypersurface. We call it a pedal height function on the hypersurface. The pedal height function is traditionally called the support function of the hypersurface with respect to the origin. We investigate, in this paper, geometric meanings of the singularities of the pedal height function. A pedal foliation is the foliation defined by the level set of the pedal height function.

On the other hand, we investigated relationships between caustics and wave front propagations as an application of the theory of graphlike Legendrian unfoldings in [11, 14]. The image of the pedal foliation by the Gauss map is considered to be a wave front propagation of a certain graphlike Legendrian unfolding (cf. §5). By applying the results in [11, 14], we

*Partially supported by Grant-in-Aid for Scientific Research (B) (22340011) and Grant-in-Aid for Challenging Exploratory Research (21654007).

†Partially supported by Grant-in-Aid for Young Scientists (B) (23740041).

2010 Mathematics Subject classification. 57R45, 58Kxx.

Key Words and Phrases. Pedal foliations, Gauss map, Lagrangian singularity, Legendrian singularity. 
obtain a new interpretation of the singularity of the Gauss map from the view point of the theory of wave front propagations (cf. $\S 6$ ). In $\S 4$, we briefly review the essential part of the theories of Lagrangian singularities and graphlike Legendrian unfoldings which we use in this paper. Especially, we give a correct proof of Proposition 4.1 in [14], which is one of the key propositions in the theory of graphlike Legendrian unfoldings (Proposition 4.3). In $\S 6$ we focus on the case for surfaces in $\mathbb{R}^{3}$. We give a classification of the surface with the constant pedal height function (i.e., the most degenerate case). Moreover, we give extra new conditions which characterize cusps of Gauss maps (cf. [2]).

We shall assume throughout the whole paper that all maps and manifolds are $C^{\infty}$ unless the contrary is explicitly stated.

\section{Hypersurfaces in Euclidean space}

In this section we review the classical theory of differential geometry on hypersurfaces in Euclidean space and introduce some singular mappings associated to geometric properties of hypersurfaces.

Let $\boldsymbol{X}: U \rightarrow \mathbb{R}^{n}$ be an embedding, where $U \subset \mathbb{R}^{n-1}$ is an open subset. We denote that $M=\boldsymbol{X}(U)$ and identify $M$ and $U$ through the embedding $\boldsymbol{X}$. The tangent space of $M$ at $p=\boldsymbol{X}(u)$ is

$$
T_{p} M=\left\langle\boldsymbol{X}_{u_{1}}(u), \boldsymbol{X}_{u_{2}}(u), \ldots, \boldsymbol{X}_{u_{n-1}}(u)\right\rangle_{\mathbb{R}}
$$

For any $\boldsymbol{a}_{1}, \boldsymbol{a}_{2}, \ldots, \boldsymbol{a}_{n-1} \in \mathbb{R}^{n}$, we define

$$
\boldsymbol{a}_{1} \times \boldsymbol{a}_{2} \times \cdots \times \boldsymbol{a}_{n-1}=\left|\begin{array}{cccc}
\boldsymbol{e}_{1} & \boldsymbol{e}_{2} & \cdots & \boldsymbol{e}_{n} \\
a_{1}^{1} & a_{2}^{1} & \cdots & a_{n}^{1} \\
a_{1}^{2} & a_{2}^{2} & \cdots & a_{n}^{2} \\
\vdots & \vdots & \ddots & \vdots \\
a_{1}^{n-1} & a_{2}^{n-1} & \cdots & a_{n}^{n-1}
\end{array}\right|,
$$

where $\left\{\boldsymbol{e}_{1}, \ldots, \boldsymbol{e}_{n}\right\}$ is the canonical basis of $\mathbb{R}^{n}$ and $\boldsymbol{a}_{i}=\left(a_{1}^{i}, a_{2}^{i}, \ldots, a_{n}^{i}\right)$. It follows that we can define the unit normal vector field

$$
\boldsymbol{n}(u)=\frac{\boldsymbol{X}_{u_{1}}(u) \times \cdots \times \boldsymbol{X}_{u_{n-1}}(u)}{\left\|\boldsymbol{X}_{u_{1}}(u) \times \cdots \times \boldsymbol{X}_{u_{n-1}}(u)\right\|}
$$

along $\boldsymbol{X}: U \rightarrow \mathbb{R}^{n}$. A map $\mathbb{G}: U \rightarrow S^{n-1}$ defined by $\mathbb{G}(u)=\boldsymbol{n}(u)$ is called the Gauss map of $M=\boldsymbol{X}(U)$. Since $\boldsymbol{n}(u)$ is the unit normal vector of $S^{n-1}$, we can identify $T_{p} M$ and $T_{\boldsymbol{n}(u)} S^{n-1}$. Under this identification, the derivative of the Gauss map $d \mathbb{G}(u)$ can be interpreted as a linear transformation on the tangent space $T_{p} M$ at $p=\boldsymbol{X}(u)$. We call the linear transformation $S_{p}=-d \mathbb{G}(u): T_{p} M \rightarrow T_{p} M$ the shape operator (or Weingarten map) of $M=\boldsymbol{X}(U)$ at $p=\boldsymbol{X}(u)$. We denote the eigenvalues of $S_{p}$ by $\kappa_{i}(p)(i=1, \ldots, n-1)$ which we call principal curvatures. We call the eigenvector of $S_{p}$ the principal direction. By definition, $\kappa_{i}(p)$ is a principal curvature if and only if $\operatorname{det}\left(S_{p}-\kappa_{i}(p) I\right)=0$. The Gauss-Kronecker curvature of $M=\boldsymbol{X}(U)$ at $p=\boldsymbol{X}(u)$ is defined to be $K(p)=\operatorname{det} S_{p}=\prod_{i=1}^{n-1} \kappa_{i}(p)$.

We say that a point $p=\boldsymbol{X}(u) \in M$ is an umbilical point if $S_{p}=\kappa(p) 1_{T_{p} M}$. We also say that $M$ is totally umbilical if all points of $M$ are umbilical. Then we have the following proposition (cf. [9, page 147, Proposition 4] for $n=3$ ). For general dimensions, the proof is given by the same method as that of [9]. 
Proposition 2.1 Suppose that $M=\boldsymbol{X}(U)$ is totally umbilical, then $\kappa(p)$ is a constant $\kappa$. Under this condition, we have the following classification:

(1) If $\kappa \neq 0$, then $M$ is a part of a hypersphere.

(2) If $\kappa=0$, then $M$ is a part of a hyperplane.

In the extrinsic differential geometry, totally umbilical hypersurfaces are considered to be the model hypersurfaces in Euclidean space. Since the set $\left\{\boldsymbol{X}_{u_{1}}, \ldots, \boldsymbol{X}_{u_{n-1}}\right\}$ is linearly independent, we induce the Riemannian metric (first fundamental form) $d s^{2}=\sum_{i, j=1}^{n-1} g_{i j} d u_{i} d u_{j}$ on $M=\boldsymbol{X}(U)$, where $g_{i j}(u)=\left\langle\boldsymbol{X}_{u_{i}}(u), \boldsymbol{X}_{u_{j}}(u)\right\rangle$ for any $u \in U$. We define the second fundamental invariant by $h_{i j}(u)=\left\langle-\boldsymbol{n}_{u_{i}}(u), \boldsymbol{X}_{u_{j}}(u)\right\rangle$ for any $u \in U$. We have the following Weingarten formula:

$$
\mathbb{G}_{u_{i}}(u)=-\sum_{j=1}^{n-1} h_{i}^{j}(u) \boldsymbol{X}_{u_{j}}(u),
$$

where $\left(h_{i}^{j}(u)\right)=\left(h_{i k}(u)\right)\left(g^{k j}(u)\right)$ and $\left(g^{k j}(u)\right)=\left(g_{k j}(u)\right)^{-1}$. By the Weingarten formula, the Gauss-Kronecker curvature is given by

$$
K(p)=\frac{\operatorname{det}\left(h_{i j}(u)\right)}{\operatorname{det}\left(g_{\alpha \beta}(u)\right)} .
$$

For a hypersurface $\boldsymbol{X}: U \rightarrow \mathbb{R}^{n}$, we say that a point $u \in U$ or $p=\boldsymbol{X}(u)$ is a flat point (or, a geodesic point) if $h_{i j}(u)=0$ for all $i, j$. Therefore, $p=\boldsymbol{X}(u)$ is a flat point if and only if $p$ is an umbilical point with the vanishing principal curvature. We say that a point $p=\boldsymbol{X}(u) \in M$ is a parabolic point if $K(p)=0$.

The cylindrical pedal of $M=\boldsymbol{X}(U)$ is defined by

$$
\mathbb{C P}_{M}: U \rightarrow S^{n-1} \times \mathbb{R} ; \mathbb{C P}_{M}(u)=(\boldsymbol{n}(u),\langle\boldsymbol{X}(u), \boldsymbol{n}(u)\rangle) .
$$

We remark that $\mathbb{C P}_{M}$ is called the dual of $M=\boldsymbol{X}(U)$ (cf. [5, 7]). For a plane curve $\gamma(s)$, $\operatorname{Pe}_{\gamma}(s)=\langle\gamma(s), \boldsymbol{n}(s)\rangle \boldsymbol{n}(s)$ is called the pedal curve of $\boldsymbol{\gamma}$ (cf. [6]), so that we call $\mathbb{C P}_{M}$ the cylindrical pedal. We have the following result (cf. [12]):

Proposition 2.2 Let $M=\boldsymbol{X}(U)$ be a hypersurface in $\mathbb{R}^{n}$. Then the following are equivalent:

(1) $M$ is totally umbilical with $\kappa=0$.

(2) The Gauss map of $M=\boldsymbol{X}(U)$ is a constant map.

(3) The cylindrical pedal of $M=\boldsymbol{X}(U)$ is a point.

(4) $M$ is a part of a hyperplane.

We can easily show that a point $p=\boldsymbol{X}(u)$ is a parabolic point of $M=\boldsymbol{X}(U)$ (i.e., a singular point of the Gauss map) if and only if it is a singular point of the cylindrical pedal. Therefore we have the following proposition:

Proposition 2.3 Let $M=\boldsymbol{X}(U)$ be a hypersurface in $\mathbb{R}^{n}$. Then the following are equivalent:

(1) $p=\boldsymbol{X}(u)$ is a parabolic point of $M$ (i.e., $K(u)=0$ ).

(2) $p=\boldsymbol{X}(u)$ is a singular point of the Gauss map of $M=\boldsymbol{X}(U)$.

(3) $p=\boldsymbol{X}(u)$ is a singular point of the cylindrical pedal of $M=\boldsymbol{X}(U)$. 
The Gauss map $\mathbb{G}(u)$ is the first component of the cylindrical pedal $\mathbb{C P}_{M}(u)$. We have a natural question as follows:

Question. What kind of information are provided by the second component of the cylindrical pedal?

We define a function $h^{\pi}: U \rightarrow \mathbb{R}$ by $h^{\pi}(u)=\langle\boldsymbol{X}(u), \boldsymbol{n}(u)\rangle$. It has been called $h^{\pi}$ the support function of $M=\boldsymbol{X}(U)$ with respect to the origin. Since $h^{\pi}$ is the second component of the cylindrical pedal, we call it the pedal height function of $M=\boldsymbol{X}(U)$ here. We remark that $h^{\pi}$ is invariant under the $S O(n)$-action and not invariant under the Euclidean motions.

\section{Pedal foliations}

A pedal foliation is the foliation in $U$ (or $M=\boldsymbol{X}(U)$ ) defined by the level set of the pedal height function $h^{\pi}$. We write

$$
\mathscr{F}^{\pi}(M)=\left\{\left(h^{\pi}\right)^{-1}\left(t_{0}\right) \mid h^{\pi}\left(u_{0}\right)=t_{0} \in \mathbb{R}\right\} .
$$

as the pedal foliation and denote by $\mathcal{L}_{u_{0}}^{\pi}(M)$ the leaf through $u_{0}$ with $h^{\pi}\left(u_{0}\right)=t_{0}$. We call $\mathcal{L}_{u_{0}}^{\pi}(M)$ a pedal leaf of $M=\boldsymbol{X}(U)$ through $u_{0} \in U$. The pedal foliation might be singular in general. The singular point of the pedal foliation is a critical point of the pedal height function $h^{\pi}$.

In order to explain the critical point of the pedal height function $h^{\pi}$, we decompose $\boldsymbol{X}(u)$ into the tangent component $\boldsymbol{X}^{T}(u)$ and the normal component $\boldsymbol{X}^{\perp}(u)$. For any $p=\boldsymbol{X}(u)$, we have $\boldsymbol{X}(u)=\boldsymbol{X}^{T}(u)+\boldsymbol{X}^{\perp}(u)$ where $\boldsymbol{X}^{T}(u) \in T_{p} M$ and $\boldsymbol{X}^{\perp}(u) \in T_{p} M^{\perp}$. Then we have the following proposition.

Proposition 3.1 Let $\boldsymbol{X}: U \rightarrow \mathbb{R}^{n}$ be a hypersurface. Then $u \in U$ is a singular point of the pedal function $h^{\pi}$ if and only if $\boldsymbol{X}^{T}(u) \in \operatorname{Ker} S_{p}$.

Proof. By definition, there exist $\mu_{i}(i=1, \ldots, n-1)$ such that $\boldsymbol{X}^{T}(u)=\sum_{i=1}^{n-1} \mu_{i} \boldsymbol{X}_{u_{i}}(u)$. Since we have $\left\langle\boldsymbol{X}(u), \boldsymbol{X}_{u_{j}}(u)\right\rangle=\sum_{i=1}^{n-1} \mu_{i} g_{i j}(u)$, we have $\sum_{j=1}^{n-1} g^{k j}(u)\left\langle\boldsymbol{X}(u), \boldsymbol{X}_{u_{j}}(u)\right\rangle=\mu_{k}$. It follows that

$$
\boldsymbol{X}^{T}(u)=\sum_{i=1}^{n-1} \sum_{j=1}^{n-1} g^{i j}(u)\left\langle\boldsymbol{X}(u), \boldsymbol{X}_{u_{j}}(u)\right\rangle \boldsymbol{X}_{u_{i}}(u)
$$

By the Weingarten formula $\mathbb{G}_{u_{i}}(u)=-\sum_{j=1}^{n-1} h_{i}^{j}(u) \boldsymbol{X}_{u_{j}}(u)$, we have

$$
\begin{aligned}
\frac{\partial h^{\pi}}{\partial u_{i}}(u) & =\left\langle\boldsymbol{X}(u), \mathbb{G}_{u_{i}}(u)\right\rangle=-\sum_{j=1}^{n-1} h_{i}^{j}(u)\left\langle\boldsymbol{X}(u), \boldsymbol{X}_{u_{j}}(u)\right\rangle \\
& =-\sum_{j=1}^{n-1} \sum_{k=1}^{n-1} h_{i k}(u) g^{k j}(u)\left\langle\boldsymbol{X}(u), \boldsymbol{X}_{u_{j}}(u)\right\rangle=-\sum_{k=1}^{n-1} h_{i k}(u) \mu_{k} .
\end{aligned}
$$

Therefore, we have

$$
\sum_{i=1}^{n-1} g^{j i}(u) \frac{\partial h^{\pi}}{\partial u_{i}}(u)=-\sum_{k=1}^{n-1}\left(\sum_{i=1}^{n-1} g^{j i}(u) h_{i k}(u)\right) \mu_{k}=-\sum_{k=1}^{n-1} h_{k}^{j}(u) \mu_{k} .
$$


Thus, $\partial h^{\pi} / \partial u_{i}(u)=0$ for $i=1, \ldots, n-1$ if and only if $\sum_{k=1}^{n-1} h_{k}^{j}(u) \mu_{k}=0$ for $j=1, \ldots, n-1$. This completes the proof.

Then we have the following corollary.

Corollary 3.2 Let $p=\boldsymbol{X}(u)$ be a singular point of $h^{\pi}$. Then $\boldsymbol{X}(u)$ is a normal vector of $M$ at $p$ or $K(p)=0$ and $\boldsymbol{X}^{T}(u) \in \operatorname{Ker} S_{p}$.

Proof. If $\boldsymbol{X}^{T}(u) \neq \mathbf{0}$, then $\operatorname{Ker} S_{p} \neq \emptyset$. This means that $K(p)=0$. If $\boldsymbol{X}^{T}(u)=\mathbf{0}$, then $\boldsymbol{X}(u)=\boldsymbol{X}^{\perp}(u)$.

We can show that the pedal foliation is non-singular in generic.

Corollary 3.3 Let $\boldsymbol{X}: U \rightarrow \mathbb{R}^{n}$ be an embedding from an open region $U \subset \mathbb{R}^{n-1}$. Suppose that $p=\boldsymbol{X}(u)$ is a singular point of $h^{\pi}$ and non-geodesic point (i.e., non-flat umbilical point). Then, under a small Euclidean motion of $M=\boldsymbol{X}(U), h^{\pi}$ is non-singular at $p=\boldsymbol{X}(u)$.

Proof. By the assumption, $\operatorname{Ker} S_{p} \neq T_{p} M$. If $K(p) \neq 0$, the position vector $\boldsymbol{X}(u)$ is not a normal vector at $p=\boldsymbol{X}(u)$ under a small Euclidean motion of $M$.

Suppose that $K(p)=0$. If we rotate $M=\boldsymbol{X}(U)$ around the normal direction (i.e, fixining the direciton of $\boldsymbol{n}(u)$ ) at the point $p=\boldsymbol{X}(u)$, then $p=\boldsymbol{X}^{\perp}(u)+\boldsymbol{X}^{T}(u)$ (i.e., of course $\boldsymbol{X}^{T}(u)$ ) does not move but $\operatorname{Ker} S_{p}$ moves. Therefore, we have $\boldsymbol{X}^{T}(u) \notin \operatorname{Ker} S_{p}$ by a small Euclidean motion of $M=\boldsymbol{X}(U)$.

By the above corollary, the pedal foliation is non-singular in generic at least locally, so that we are interested in differential geometric properties of leaves.

We now consider the restriction $\mathbb{G} \mid \mathcal{L}_{u_{0}}^{\pi}(M)$ of the Gauss map $\mathbb{G}$ on the pedal leaf through $u_{0} \in U$, which is called the pedal Gauss map of $M=\boldsymbol{X}(U)$ at $u_{0} \in U$.

\section{Graphlike Legendrian unfoldings}

In order to apply the theories of Lagrangian singularities and graphlike Legendrian unfoldings, we explain the essential parts of the theories which we need in this paper. The detailed descriptions and the results are referred to be the articles $[1,11,14,22,23]$.

Firstly, we consider the cotangent bundle $\pi: T^{*} \mathbb{R}^{n} \rightarrow \mathbb{R}^{n}$. Let $(x, p)=\left(x_{1}, \ldots, x_{n}, p_{1}, \ldots, p_{n}\right)$ be the canonical coordinate on $T^{*} \mathbb{R}^{n}$. Then the canonical symplectic structure on $T^{*} \mathbb{R}^{n}$ is given by the canonical two form $\omega=\sum_{i=1}^{n} d p_{i} \wedge d x_{i}$. Let $i: L \subset T^{*} \mathbb{R}^{n}$ be a submanifold. We say that $i$ is a Lagrangian submanifold if $\operatorname{dim} L$ and $i^{*} \omega=0$. Let $F:\left(\mathbb{R}^{k} \times \mathbb{R}^{n}, 0\right) \rightarrow(\mathbb{R}, 0)$ be an $n$-parameter unfolding of function germs. We say that $F$ is a Morse family of functions if the map germ

$$
\Delta F=\left(\frac{\partial F}{\partial q_{1}}, \ldots, \frac{\partial F}{\partial q_{k}}\right):\left(\mathbb{R}^{k} \times \mathbb{R}^{n}, 0\right) \rightarrow\left(\mathbb{R}^{k}, 0\right)
$$

is a non-singular, where $(q, x)=\left(q_{1}, \ldots, q_{k}, x_{1}, \ldots, x_{n}\right) \in\left(\mathbb{R}^{k} \times \mathbb{R}^{n}, 0\right)$. In this case, we have a smooth $n$-dimensional submanifold germ $C(F)=(\Delta F)^{-1}(0) \subset\left(\mathbb{R}^{k} \times \mathbb{R}^{n}, 0\right)$ and a map germ $L(F):(C(F), 0) \rightarrow T^{*} \mathbb{R}^{n}$ defined by

$$
L(F)(q, x)=\left(x, \frac{\partial F}{\partial x_{1}}(q, x), \ldots, \frac{\partial F}{\partial x_{n}}(q, x)\right) .
$$


We can show that $L(F)(C(F))$ is a Lagrangian submanifold germ. We say that $F$ is a generating family of $L(F)(C(F))$.

We now define an equivalence relation among Lagrangian submanifold germs. Let $F, G$ : $\left(\mathbb{R}^{k} \times \mathbb{R}^{n}, 0\right) \rightarrow(\mathbb{R}, 0)$ be Morse families of functions. Then the Lagrangian submanifold germs $(L(F)(C(F)), \mathbf{0})$ and $(L(G)(C(G)), \mathbf{0})$ are said to be Lagrangian equivalent if there exist a symplectic diffeomorphism germ $\hat{\tau}:\left(T^{*} \mathbb{R}^{n}, p\right) \rightarrow\left(T^{*} \mathbb{R}^{n}, p^{\prime}\right)$ and a diffeomorphism germ $\tau$ : $\left(\mathbb{R}^{n}, \pi(p)\right) \rightarrow\left(\mathbb{R}^{n}, \pi\left(p^{\prime}\right)\right)$ such that $\hat{\tau}(L(F)(C(F))=L(G)(C(G))$ and $\pi \circ \hat{\tau}=\tau \circ \pi$, where $\hat{\tau}$ is a symplectic diffeomorphism germ if $\hat{\tau}^{*} \omega=\omega$. By using the Lagrangian equivalence, we can define the notion of Lagrangian stability for Lagrangian submanifold germs by the ordinary way (see, $[1$, Part III]).

We can interpret the Lagrangian equivalence by using the notion of generating families. Let $\mathcal{E}_{x}$ be the ring of function germs of $x=\left(x_{1}, \ldots, x_{n}\right)$ variables at the origin. Let $F, G$ : $\left(\mathbb{R}^{k} \times \mathbb{R}^{n}, 0\right) \rightarrow(\mathbb{R}, 0)$ be function germs. We say that $F$ and $G$ are $P$ - $\mathcal{R}^{+}$-equivalent if there exist a diffeomorphism germ $\Phi:\left(\mathbb{R}^{k} \times \mathbb{R}^{n}, 0\right) \rightarrow\left(\mathbb{R}^{k} \times \mathbb{R}^{n}, 0\right)$ of the form $\Phi(q, x)=\left(\phi_{1}(q, x), \phi_{2}(x)\right)$ and a function germ $h:\left(\mathbb{R}^{n}, 0\right) \rightarrow(\mathbb{R}, 0)$ such that $G(q, x)=F(\Phi(q, x))+h(x)$. For any $F_{1}:\left(\mathbb{R}^{k} \times \mathbb{R}^{n}, 0\right) \rightarrow(\mathbb{R}, 0)$ and $F_{2}:\left(\mathbb{R}^{k^{\prime}} \times \mathbb{R}^{n}, 0\right) \rightarrow(\mathbb{R}, 0)$, Let $F:\left(\mathbb{R}^{k} \times \mathbb{R}^{n}, 0\right) \rightarrow(\mathbb{R}, 0)$ be a function germ. We say that $F$ is an $\mathcal{R}^{+}$-versal deformation of $f=\left.F\right|_{\mathbb{R}^{k} \times\{0\}}$ if

$$
\mathcal{E}_{q}=J_{f}+\left\langle\frac{\partial F}{\partial x_{1}}\left|\mathbb{R}^{k} \times\{0\}, \ldots, \frac{\partial F}{\partial x_{n}}\right| \mathbb{R}^{k} \times\{0\}\right\rangle_{\mathbb{R}}+\langle 1\rangle_{\mathbb{R}},
$$

where

$$
J_{f}=\left\langle\frac{\partial f}{\partial q_{1}}(q), \ldots, \frac{\partial f}{\partial q_{k}}(q)\right\rangle_{\mathcal{E}_{q}} .
$$

Then we have the following theorem[1, page 304 and 325]:

Theorem 4.1 Let $F:\left(\mathbb{R}^{k} \times \mathbb{R}^{n}, 0\right) \rightarrow(\mathbb{R}, 0)$ and $G:\left(\mathbb{R}^{k} \times \mathbb{R}^{n}, 0\right) \rightarrow(\mathbb{R}, 0)$ be Morse families of functions. Then we have the following:

(1) $L(F)(C(F))$ and $L(G)(C(G))$ are Lagrangian equivalent if and only if $F$ and $G$ are $P-\mathcal{R}^{+}$equivalent.

(2) $L(F)(C(F))$ is a Lagrange stable if and only if $F$ is an $\mathcal{R}^{+}$-versal deformation of $f$.

In [1], the assertion (1) of the above theorem is a slightly different. It is used the notion of stable $P$ - $\mathcal{R}^{+}$-equivalences among Morse families. However, the above assertion is enough for our situation.

Secondly, we now give a brief review on the theory of graphlike Legendrian unfoldings. The notion of graphlike Legendrian unfoldings is defined in the projective cotangent bundle $\bar{\pi}: P T^{*}\left(\mathbb{R}^{n} \times \mathbb{R}\right) \rightarrow \mathbb{R}^{n} \times \mathbb{R}$ (cf. [11]). We remark that the affine open subset $U_{\tau}=\{((x, t),[\xi:$ $\tau]) \mid \tau \neq 0\}$ of $P T^{*}\left(\mathbb{R}^{n} \times \mathbb{R}\right)$ is canonically identified with the 1 -jet space $J^{1}\left(\mathbb{R}^{n}, \mathbb{R}\right)$, see in $[11,14]$. For a Morse family of functions $F:\left(\mathbb{R}^{k} \times \mathbb{R}^{n}, 0\right) \rightarrow(\mathbb{R}, 0)$, we define a map $\mathfrak{L}_{F}:(C(F), 0) \rightarrow$ $J^{1}\left(\mathbb{R}^{n}, \mathbb{R}\right)$ by

$$
\mathfrak{L}_{F}(q, x)=\left(x, F(q, x), \frac{\partial F}{\partial x_{1}}(q, x), \ldots, \frac{\partial F}{\partial x_{n}}(q, x)\right) .
$$

Then $\left(\mathfrak{L}_{F}(C(F)), \mathbf{0}\right)$ is a Legendrian submanifold germ which is called a graphlike Legendrian unfolding. We call the set germ $W\left(\mathfrak{L}_{F}\right)=\bar{\pi}\left(\mathfrak{L}_{F}(C(F))\right.$ the graphlike wave front of $\mathfrak{L}_{F}(C(F))$. A graphlike Legendrian unfolding $\left(\mathfrak{L}_{F}(C(F)), \mathbf{0}\right)$ is said to be non-degenerate if $F \mid C(F)$ is non-singular. We say that $F$ is a generating family of the graphlike Legendrian unfolding 
$\mathfrak{L}_{F}(C(F))$. We can use all equivalence relations introduced in the previous paper $[13,14,15]$. Especially, the $S . P^{+}$-Legendrian equivalence among graphlike Legendrian unfoldings was given in the above contects. Since we do not need the definition here, we omit to give the definition (see [13]). We also consider the stability of graphlike Legendrian unfolding with respect to $S . P^{+}$ -Legendrian equivalence which is analogous to the stability of Lagrangian submanifold germs with respect to Lagrangian equivalence (cf. [1, Part III]). We denote that $\bar{F}(q, x, t)=F(q, x)-t$ and $\bar{f}(q, t)=f(q)-t$ for $f(q)=F(q, 0)$. We can represent the extended tangent space of $\bar{f}:\left(\mathbb{R}^{k} \times \mathbb{R}, 0\right) \rightarrow(\mathbb{R}, 0)$ relative to $S . P^{+}-\mathcal{K}$ by

$$
T_{e}\left(S . P^{+}-\mathcal{K}\right)(\bar{f})=\left\langle\frac{\partial f}{\partial q_{1}}(q), \ldots, \frac{\partial f}{\partial q_{k}}(q), f(q)-t\right\rangle_{\mathcal{E}_{(q, t)}}+\langle 1\rangle_{\mathbb{R}} .
$$

For an unfolding $\bar{F}:\left(\mathbb{R}^{k} \times \mathbb{R}^{n}, 0\right) \rightarrow(\mathbb{R}, 0)$ of $f, \bar{F}$ is $S . P^{+}-\mathcal{K}$-versal deformation of $\bar{f}$ if

$$
\mathcal{E}_{(q, t)}=T_{e}\left(S . P^{+}-\mathcal{K}\right)(\bar{f})+\left\langle\frac{\partial F}{\partial x_{1}}\left|\mathbb{R}^{k} \times\{0\}, \ldots, \frac{\partial F}{\partial x_{n}}\right| \mathbb{R}^{k} \times\{0\}\right\rangle_{\mathbb{R}} .
$$

Then we have the following theorem $[11,14,23]$.

Theorem 4.2 Let $F:\left(\mathbb{R}^{k} \times \mathbb{R}^{n}, 0\right) \rightarrow(\mathbb{R}, 0)$ be a Morse family of functions. Then $\mathcal{L}_{F}(C(F))$ is $S . P^{+}$-Legendre stable if and only if $\bar{F}$ is a $S . P^{+}-\mathcal{K}$-versal deformation of $\bar{f}$.

We gave a proof of the following proposition in [14]. However, there are some gaps on the arguments of the proof. Here we give a correct proof of Proposition 4.1 in [14].

Proposition 4.3 Let $F:\left(\mathbb{R}^{k} \times \mathbb{R}^{n}, 0\right) \rightarrow(\mathbb{R}, 0)$ be a Morse family of functions. If $\mathfrak{L}_{F}(C(F))$ is a S.P $P^{+}$-Legendre stable, then $L(F)(C(F))$ is a Lagrange stable.

Proof. Since $\mathfrak{L}_{F}(C(F))$ is a $S . P^{+}$-Legendre stable,

$$
\operatorname{dim}_{\mathbb{R}} \frac{\mathcal{E}_{(q, t)}}{\left\langle\frac{\partial f}{\partial q_{1}}(q), \ldots, \frac{\partial f}{\partial q_{k}}(q), f(q)-t\right\rangle_{\mathcal{E}_{(q, t)}}+\langle 1\rangle_{\mathbb{R}}}<\infty .
$$

It follows that $\operatorname{dim}_{\mathbb{R}} \mathcal{E}_{q} /\left\langle\frac{\partial f}{\partial q_{1}}(q), \ldots, \frac{\partial f}{\partial q_{k}}(q), f(q)\right\rangle_{\mathcal{E}_{q}}<\infty$, namely, $f$ is a $\mathcal{K}$-finitely determined (see the definition $[8,18]$ ). It is a well-known result that $f$ is a $\mathcal{K}$-finitely determined if and only if $f$ is an $\mathcal{R}^{+}$-finitely determined, see [8]. Under the condition that $f$ is an $\mathcal{R}^{+}$-finitely determined, $F$ is an $\mathcal{R}^{+}$-versal deformation of $f$ if and only if $F$ is an $\mathcal{R}^{+}$-transversal deformation of $f$, namely, there exists a number $\ell \in \mathbb{N}$ such that

$$
\mathcal{E}_{q}=J_{f}+\left\langle\frac{\partial F}{\partial x_{1}}\left|\mathbb{R}^{k} \times\{0\}, \ldots, \frac{\partial F}{\partial x_{n}}\right| \mathbb{R}^{k} \times\{0\}\right\rangle_{\mathbb{R}}+\langle 1\rangle_{\mathbb{R}}+\mathcal{M}_{q}^{\ell+1} .
$$

Hence it is enough to show the equality $(1)$ by Theorem 4.1. Let $g(q) \in \mathcal{E}_{q}$. Since $g(q) \in \mathcal{E}_{(q, t)}$, there exist $\lambda_{i}(q, t), \mu(q, t) \in \mathcal{E}_{(q, t)}(i=1, \ldots, k)$ and $c, c_{j} \in \mathbb{R}(j=1, \ldots, n)$ such that

$$
g(q)=\sum_{i=1}^{k} \lambda_{i}(q, t) \frac{\partial f}{\partial q_{i}}(q)+\mu(q, t)(f(q)-t)+c+\sum_{j=1}^{n} c_{j} \frac{\partial F}{\partial x_{j}}(q, 0) .
$$


Differentiating the equality (2) with respect to $t$, we have

$$
0=\sum_{i=1}^{k} \frac{\partial \lambda_{i}}{\partial t}(q, t) \frac{\partial f}{\partial q_{i}}(q)+\frac{\partial \mu}{\partial t}(q, t)(f(q)-t)-\mu(q, t)
$$

We put $t=0$ in $(3), 0=\sum_{i=1}^{k}\left(\partial \lambda_{i} / \partial t\right)(q, 0)\left(\partial f / \partial q_{i}\right)(q)+(\partial \mu / \partial t)(q, 0) f(q)-\mu(q, 0)$. Also we put $t=0$ in $(2)$, then

$$
\begin{aligned}
g(q) & =\sum_{i=1}^{k} \lambda_{i}(q, 0) \frac{\partial f}{\partial q_{i}}(q)+\mu(q, 0) f(q)+c+\sum_{j=1}^{n} c_{j} \frac{\partial F}{\partial x_{j}}(q, 0) \\
& =\sum_{i=1}^{k} \alpha_{i}(q) \frac{\partial f}{\partial q_{i}}(q)+\frac{\partial \mu}{\partial t}(q, 0) f^{2}(q)+c+\sum_{j=1}^{n} c_{j} \frac{\partial F}{\partial x_{j}}(q, 0),
\end{aligned}
$$

for some $\alpha_{i} \in \mathcal{E}_{q}, i=1 \ldots, k$. Again differentiating (3) with respect to $t$ and put $t=0$, then

$$
0=\sum_{i=1}^{k} \frac{\partial^{2} \lambda_{i}}{\partial t^{2}}(q, 0) \frac{\partial f}{\partial q_{i}}(q)+\frac{\partial^{2} \mu}{\partial t^{2}}(q, 0) f(q)-2 \frac{\partial \mu}{\partial t}(q, 0)
$$

Hence (4) is equal to

$$
\sum_{i=1}^{k} \beta_{i}(q) \frac{\partial f}{\partial q_{i}}(q)+\frac{1}{2} \frac{\partial^{2} \mu}{\partial t^{2}}(q, 0) f^{3}(q)+c+\sum_{j=1}^{n} c_{j} \frac{\partial F}{\partial x_{j}}(q, 0)
$$

for some $\beta_{i} \in \mathcal{E}_{q}, i=1, \ldots, k$. Inductively, we take $\ell$-times differentiate (3) with respect to $t$ and put $t=0$, then we have

$$
g(q)=\sum_{i=1}^{k} \gamma_{i}(q) \frac{\partial f}{\partial q_{i}}(q)+\frac{1}{\ell !} \frac{\partial^{\ell} \mu}{\partial t^{\ell}}(q, 0) f^{\ell+1}(q)+c+\sum_{j=1}^{n} c_{j} \frac{\partial F}{\partial x_{j}}(q, 0),
$$

for some $\gamma_{i} \in \mathcal{E}_{q}, i=1, \ldots, k$. It follows that $g(q)$ is contained in the right hand of (1). This completes the proof.

We consider a relationship of the equivalence relations between Lagrangian immersion germs and corresponding graphlike Legendrian unfoldings. Let $\mathfrak{L}_{F}:(C(F), 0) \rightarrow\left(J^{1}\left(\mathbb{R}^{n}, \mathbb{R}\right), p_{0}\right)$ and $\mathfrak{L}_{G}:(C(G), 0) \rightarrow\left(J^{1}\left(\mathbb{R}^{n}, \mathbb{R}\right), q_{0}\right)$ be graphlike Legendrian unfolding germs. We say that graphlike wave fronts $W\left(\mathfrak{L}_{F}\right)$ and $W\left(\mathfrak{L}_{G}\right)$ are $S . P^{+}$-diffeomorphic if there exists a diffeomorphism germ $\Phi:\left(\mathbb{R}^{n} \times \mathbb{R}, \bar{\pi}\left(p_{0}\right)\right) \rightarrow\left(\mathbb{R}^{n} \times \mathbb{R}, \bar{\pi}\left(q_{0}\right)\right)$ of the form $\Phi(x, t)=\left(\phi_{1}(x), t+\alpha(x)\right)$ such that $\Phi\left(W\left(\mathfrak{L}_{F}\right)\right)=W\left(\mathfrak{L}_{G}\right)$. Then we have the following result:

Theorem 4.4 ([14]) Suppose that $L(F)(C(F))$ and $L(G)(C(G))$ are Lagrange stable. Then Lagrangian submanifold germs $L(F)(C(F))$ and $L(G)(C(G))$ are Lagrangian equivalent if and only if graphlike wave fronts $W\left(\mathfrak{L}_{F}\right)$ and $W\left(\mathfrak{L}_{G}\right)$ are $S . P^{+}$-diffeomorphic. 


\section{$5 \quad$ Height functions}

We respectively define two functions

$$
H: U \times S^{n-1} \rightarrow \mathbb{R}
$$

by $H(u, \boldsymbol{v})=\langle\boldsymbol{X}(u), \boldsymbol{v}\rangle$ and

$$
\widetilde{H}: U \times\left(S^{n-1} \times \mathbb{R}\right) \rightarrow \mathbb{R}
$$

by $\widetilde{H}(u,(\boldsymbol{v}, t))=H(u, \boldsymbol{v})-t=\langle\boldsymbol{X}(u), \boldsymbol{v}\rangle-t$. We call $H$ a family of height functions and $\widetilde{H}$ a family of extended height functions of $M=\boldsymbol{X}(U)$. We denote that $h_{\boldsymbol{v}}(u)=H(u, \boldsymbol{v})$ and $\widetilde{h}_{(\boldsymbol{v}, t)}(u)=\widetilde{H}(u,(\boldsymbol{v}, t))$. By the straightforward calculations, we can show the following proposition:

Proposition 5.1 Let $M=\boldsymbol{X}(U)$ be a hypersurface in $\mathbb{R}^{n}$. Then

(1) $\left(\partial h_{\boldsymbol{v}} / \partial u_{i}\right)(u)=0(i=1, \ldots, n-1)$ if and only if $\boldsymbol{v}= \pm \boldsymbol{n}(u)$.

(2) $\widetilde{h}_{(\boldsymbol{v}, t)}(u)=\left(\partial \widetilde{h}_{(\boldsymbol{v}, t)} / \partial u_{i}\right)(u)=0(i=1, \ldots, n-1)$ if and only if $(\boldsymbol{v}, t)= \pm(\boldsymbol{n}(u),\langle\boldsymbol{n}(u), \boldsymbol{X}(u)\rangle)$.

For $\boldsymbol{v}=\mathbb{G}(u)$, we have

$$
\frac{\partial^{2} H}{\partial u_{i} \partial u_{j}}(u, \boldsymbol{v})=\left\langle\boldsymbol{X}_{u_{i} u_{j}}(u), \boldsymbol{v}\right\rangle=-\left\langle\boldsymbol{X}_{u_{i}}(u), \boldsymbol{n}_{u_{j}}(u)\right\rangle=h_{i j}(u) \text {. }
$$

Therefore, for any $\boldsymbol{v}=\mathbb{G}(u)$, $\operatorname{det}\left(\mathcal{H}\left(h_{v}\right)(u)\right)=\operatorname{det}\left(\left(\partial^{2} H / \partial u_{i} \partial u_{j}\right)(u, \boldsymbol{v})\right)=0$ if and only if $K(p)=0$ (i.e., $p=\boldsymbol{X}(u)$ is a parabolic point), where $\mathcal{H}\left(h_{v}\right)(u)$ is the Hessian matrix of $h_{v}$ at a point $u$. By the above calculation, we have the following results [12]:

Proposition 5.2 For any $p=\boldsymbol{X}(u)$, we have the following assertions:

Suppose that $\boldsymbol{v}=\mathbb{G}(u)$, then

(1) $p$ is a parabolic point if and only if $\operatorname{det}\left(\mathcal{H}\left(h_{v}\right)(u)\right)=0$.

(2) $p$ is a flat point if and only if $\operatorname{rank} \mathcal{H}\left(h_{v}\right)(u)=0$.

We now consider the relationship with the theories of Lagrangian singularities and graphlike Legendrian unfoldings. By [12, Proposition 4.1], we have the following proposition.

Proposition 5.3 Let $\boldsymbol{X}: U \rightarrow M$ be an embedding.

(1) The family of height functions $H: U \times S^{n-1} \rightarrow \mathbb{R}$ of $M=\boldsymbol{X}(U)$ is a Morse family of functions.

(2) The family of extended height functions $\widetilde{H}: U \times\left(S^{n-1} \times \mathbb{R}\right) \rightarrow \mathbb{R}$ of $M=\boldsymbol{X}(U)$ is a graphlike Morse family of hypersurfaces.

By the arguments in $\S 4$, we have a graphlike Legendrian unfolding whose generating family is the height function of $M=\boldsymbol{X}(U)$. By Proposition 5.1, we have

$$
C(H)=\left\{(u, \pm \boldsymbol{n}(u)) \in U \times S^{n-1} \mid u \in U\right\} .
$$

It follows that we have a graphlike Legendrian unfolding $\mathfrak{L}_{H}: C(H) \rightarrow T^{*} S^{n-1} \times \mathbb{R} \cong$ $J^{1}\left(S^{n-1}, \mathbb{R}\right)$ defined by

$$
\mathfrak{L}_{H}(u, \pm \boldsymbol{n}(u))=(L(H)(u, \pm \boldsymbol{n}(u)),\langle \pm \boldsymbol{n}(u), \boldsymbol{X}(u)\rangle),
$$

where $L(H): C(H) \rightarrow T^{*} S^{n-1}$ is the corresponding Lagrangian immersion. By definition, we have the following corollary of the above proposition: 
Corollary 5.4 Under the above notations, $\mathfrak{L}_{H}(C(H))$ is a graphlike Legendrian unfolding such that the height function $H: U \times S^{n-1} \rightarrow \mathbb{R}$ of $M=\boldsymbol{X}(U)$ is a generating family of $\mathfrak{L}_{H}(C(H)$ ).

By Corollary 5.4 and Proposition 5.1, we have the graphlike Legendrian unfolding $\mathfrak{L}_{H}(C(H))$ whose graphlike wave front is the cylindrical pedal $\pm \mathbb{C P}_{M}$ of $M=\boldsymbol{X}(U)$. We call $\mathfrak{L}_{H}(C(H))$ the Legendrian lift of the cylindrical pedal $\mathbb{C P}_{M}$ of $M=\boldsymbol{X}(U)$. By definition, we have $H(u, \pm \boldsymbol{n}(u))= \pm\langle\boldsymbol{X}(u), \boldsymbol{n}(u)\rangle= \pm h^{\pi}(u)$. Therefore, we have the following proposition.

Proposition 5.5 The restriction of the height function $H \mid C(H)$ is non-singular at $u \in U$ if and only if the pedal height function $h^{\pi}$ is non-singular at $u \in U$.

It follows that the graphlike Legendrian unfolding $\mathfrak{L}_{H}(C(H))$ is non-degenerate if and only if the pedal height function $h^{\pi}$ is non-singular.

\section{$6 \quad$ Families of wave fronts induced by Gauss maps}

In this section, we consider general geometric properties of singularities of the pedal foliation of a hypersurface in Euclidean space. Let $\mathscr{F}^{\pi}(M)$ be the pedal foliation on a hypersurface $M=\boldsymbol{X}(U)$. Suppose that $p=\boldsymbol{X}\left(u_{0}\right) \in M$ is a non-singular point of the pedal height function $h^{\pi}$, so that the germ of the pedal foliation $\left(\mathscr{F}^{\pi}(M), p\right)$ is non-singular. We call the germ of the pedal leaf $\mathcal{L}_{u_{0}}^{\pi}(M)$ through $p$ the central pedal leaf of the pedal foliation germ $\left(\mathscr{F}^{\pi}(M), p\right)$. We consider the family of pedal Gauss map germs $\left\{\mathbb{G} \mid \mathcal{L}_{u}^{\pi}(M)\right\}_{h^{\pi}(u) \in\left(\mathbb{R}, h^{\pi}\left(u_{0}\right)\right)}$. Let $\pi_{1}: S^{n-1} \times \mathbb{R} \rightarrow$ $S^{n-1}$ and $\pi_{2}: S^{n-1} \times \mathbb{R} \rightarrow \mathbb{R}$ be the canonical projections. Then $\mathbb{G}\left(\mathcal{L}_{u}^{\pi}(M)\right)=\pi_{1}\left(\pi_{2}^{-1}(t) \cap \mathbb{C P}_{M}\right)$ for each $t \in\left(\mathbb{R}, h^{\pi}\left(u_{0}\right)\right)$ is the small front of the non-degenerate graphlike Legendrian unfolding $\mathfrak{L}_{H}(C(H))$. Thus, the family of the image of pedal Gauss map germs $\left\{\mathbb{G} \mid \mathcal{L}_{u}^{\pi}(M)\right\}_{h^{\pi}(u) \in\left(\mathbb{R}, h^{\pi}\left(u_{0}\right)\right)}$ is a family of wave fronts corresponding to the graphlike Legendrian unfolding $\mathfrak{L}_{H}(C(H))$. We can apply the theory of graphlike Legendrian unfoldings.

On the other hand, in order to understand the geometric meaning of singularities of Gauss maps (or equivalently, cylindrical pedal), we review the theory of contact of submanifolds with foliations $[10,13,14]$. Let $X_{i}(i=1,2)$ be submanifolds of $\mathbb{R}^{n}$ with $\operatorname{dim} X_{1}=\operatorname{dim} X_{2}$, $g_{i}:\left(X_{i}, \bar{x}_{i}\right) \rightarrow\left(\mathbb{R}^{n}, \bar{y}_{i}\right)$ be immersion germs and $f_{i}:\left(\mathbb{R}^{n}, \bar{y}_{i}\right) \rightarrow(\mathbb{R}, 0)$ be submersion germs. For a submersion germ $f:\left(\mathbb{R}^{n}, 0\right) \rightarrow(\mathbb{R}, 0)$, we denote that $\mathcal{F}(f)$ is the regular foliation defined by $f$; i.e., $\mathcal{F}(f)=\left\{f^{-1}(c) \mid c \in(\mathbb{R}, 0)\right\}$. We say that the contact of $X_{1}$ with the regular foliation $\mathcal{F}\left(f_{1}\right)$ at $\bar{y}_{1}$ is the same type as the contact of $X_{2}$ with the regular foliation $\mathcal{F}\left(f_{2}\right)$ at $\bar{y}_{2}$ if there is a diffeomorphism germ $\Phi:\left(\mathbb{R}^{n}, \bar{y}_{1}\right) \rightarrow\left(\mathbb{R}^{n}, \bar{y}_{2}\right)$ such that $\Phi\left(X_{1}\right)=X_{2}$ and $\Phi\left(Y_{1}(c)\right)=Y_{2}(c)$ for each $c \in(\mathbb{R}, 0)$, where $Y_{i}(c)=f_{i}^{-1}(c)$. In this case we write

$$
K\left(X_{1}, \mathcal{F}\left(f_{1}\right) ; \bar{y}_{1}\right)=K\left(X_{2}, \mathcal{F}\left(f_{2}\right) ; \bar{y}_{2}\right) .
$$

We apply the method of Goryunov [10, Appendix] to the case for $\mathcal{R}^{+}$-equivalence among function germs, so that we have the following:

Proposition 6.1 ([13]) Let $X_{i}(i=1,2)$ be submanifolds of $\mathbb{R}^{n}$ with $\operatorname{dim} X_{1}=\operatorname{dim} X_{2}-1$, $g_{i}:\left(X_{i}, \bar{x}_{i}\right) \rightarrow\left(\mathbb{R}^{n}, \bar{y}_{i}\right)$ be immersion germs and $f_{i}:\left(\mathbb{R}^{n}, \bar{y}_{i}\right) \rightarrow(\mathbb{R}, 0)$ be submersion germs. We assume that $\bar{x}_{i}$ are singularities of function germs $f_{i} \circ g_{i}:\left(X_{i}, \bar{x}_{i}\right) \rightarrow(\mathbb{R}, 0)$. Then $K\left(X_{1}, \mathcal{F}\left(f_{1}\right) ; \bar{y}_{1}\right)=K\left(X_{2}, \mathcal{F}\left(f_{2}\right) ; \bar{y}_{2}\right)$ if and only if $f_{1} \circ g_{1}$ and $f_{2} \circ g_{2}$ are $\mathcal{R}^{+}$-equivalent.

We consider a function $\mathfrak{H}: \mathbb{R}^{n} \times S^{n-1} \rightarrow \mathbb{R}$ defined by $\mathfrak{H}(\boldsymbol{x}, \boldsymbol{v})=\langle\boldsymbol{x}, \boldsymbol{v}\rangle$. For a hypersurface $\boldsymbol{X}: U \rightarrow \mathbb{R}^{n}$, we have $H=\mathfrak{H} \circ\left(\boldsymbol{X} \times 1_{S^{n-1}}\right)$. We denote $\mathfrak{h}_{\boldsymbol{v}}(\boldsymbol{x})=\mathfrak{H}(\boldsymbol{x}, \boldsymbol{v})$ for $\boldsymbol{v} \in S^{n-1}$. Suppose 
that $\boldsymbol{v}_{0}=\boldsymbol{n}\left(u_{0}\right)$ and $t_{0}=h^{\pi}\left(u_{0}\right)=\left\langle\boldsymbol{X}\left(u_{0}\right), \boldsymbol{v}_{0}\right\rangle$. By Proposition 5.1, $\mathfrak{h}_{\boldsymbol{v}_{0}}^{-1}\left(t_{0}\right)$ is tangent to $M$ at $p_{0}=\boldsymbol{X}\left(u_{0}\right)$. We denote the tangent hyperplane of $M$ at $p_{0}$ by $T M_{p_{0}}$. Then $\mathfrak{h}_{\boldsymbol{v}_{0}}^{-1}(t)$ for $t \in\left(t_{0}-\varepsilon, t_{0}+\varepsilon\right)$ is a hyperplane parallel to $T M_{p_{0}}$. Therefore, we have a foliation $\mathcal{P} \mathcal{F}\left(T M_{p_{0}}\right)$ consists of the family of hyperplane parallel to $T M_{p_{0}}$ :

$$
\mathcal{P F}\left(T M_{p_{0}}\right)=\left\{\mathfrak{h}_{\boldsymbol{v}_{0}}^{-1}(t) \mid t \in\left(t_{0}-\varepsilon, t_{0}+\varepsilon\right)\right\} .
$$

We call $\mathcal{P} \mathcal{F}\left(T M_{p_{0}}\right)$ the foliation of parallel tangent hyperplanes of $M$ at $p_{0}$

We now give a characterization of singularities of Gauss maps and cylindrical pedals. Let $\boldsymbol{X}^{i}:\left(U^{i}, u^{i}\right) \rightarrow\left(\mathbb{R}^{n}, p^{i}\right),(i=1,2)$ be hypersurface germs and $H^{i}:\left(U^{i} \times S^{n-1},\left(u^{i}, \boldsymbol{v}^{i}\right)\right) \rightarrow \mathbb{R}$ be families of height functions on $M^{i}=\boldsymbol{X}^{i}\left(U^{i}\right)$, where $\boldsymbol{v}^{i}=\mathbb{G}^{i}\left(u^{i}\right),(i=1,2)$. Then we have Lagrangian submanifold germs $L\left(H^{i}\right)\left(C\left(H^{i}\right)\right)$ in $T^{*} S^{n-1}$ which cover the Gauss maps $\mathbb{G}^{i}$ as Lagrangian maps. We also have the graphlike Legendrian unfoldings $\mathfrak{L}_{H^{i}}\left(C\left(H^{i}\right)\right)$ whose graphlike wavefronts are the cylindrical pedals $\mathbb{C P}_{M^{i}}\left(U^{i}\right)$.

Theorem 6.2 Suppose that the Lagrange submanifold germs $L\left(H^{i}\right)\left(C\left(H^{i}\right)\right)$ are Lagrangian stable. Then the following conditions are equivalent:

(1) $L\left(H^{1}\right)\left(C\left(H_{1}\right)\right)$ and $L\left(H^{2}\right)\left(C\left(H_{2}\right)\right)$ are Lagrangian equivalent,

(2) $\left(\mathbb{C P}_{M^{1}}\left(U^{1}\right),\left(\boldsymbol{v}^{1}, t^{1}\right)\right)$ and $\left(\mathbb{C P}_{M^{2}}\left(U^{2}\right),\left(\boldsymbol{v}^{2}, t^{2}\right)\right)$ are $S . P^{+}-\mathcal{K}$-diffeomorphic,

(3) $\mathfrak{L}_{H^{1}}\left(C\left(H^{1}\right)\right)$ and $\mathfrak{L}_{H^{2}}\left(C\left(H^{2}\right)\right)$ are $S . P^{+}$-Legendrian equivalent,

(4) $K\left(M^{1}, \mathcal{P F}\left(T M_{p^{1}}^{1}\right) ; p^{1}\right)=K\left(M^{2}, \mathcal{P} \mathcal{F}\left(T M_{p^{2}}^{2}\right) ; p^{2}\right)$.

Proof. By Proposition 6.1, the conditions (4) is equivalent to the condition that the height function germs $h_{\boldsymbol{v}^{1}}^{1}$ and $h_{\boldsymbol{v}^{2}}^{1}$ are $\mathcal{R}^{+}$-equivalent. By the assumption and Theorem $4.1, H^{i}$ is an $\mathcal{R}^{+}$-versal unfolding of $h_{\boldsymbol{v}^{i}}^{i}$ as germs. Then the uniqueness theorem of the $\mathcal{R}^{+}$-versal unfoldings (cf. $[4,18]$ ) asserts that $h_{\boldsymbol{v}^{1}}^{1}$ and $h_{\boldsymbol{v}^{2}}^{1}$ are $\mathcal{R}^{+}$-equivalent if and only if $H^{1}$ and $H^{2}$ are $P$ - $\mathcal{R}^{+}$equivalent. The last condition is equivalent to the condition (1) by Theorem 4.1. Since the cylindrical pedal is the graphlike wave front of the graphlike Legendrian unfolding generated by the family of height functions, the conditions (1) and (2) are equivalent by Theorem 4.4. Moreover, by [14, Proposition 3.5], the conditions (2) and (3) are equivalent. This completes the proof.

We remark that the condition (1) of the above theorem implies that the corresponding Gauss maps $\mathbb{G}^{1}$ and $\mathbb{G}^{2}$ are $\mathcal{A}$-equivalent. Here two map germs $f, g:\left(\mathbb{R}^{n}, \mathbf{0}\right) \rightarrow\left(\mathbb{R}^{p}, \mathbf{0}\right)$ are said to be $\mathcal{A}$ equivalent if there exist diffeomorphism germs $\phi:\left(\mathbb{R}^{n}, \mathbf{0}\right) \rightarrow\left(\mathbb{R}^{n}, \mathbf{0}\right)$ and $\psi:\left(\mathbb{R}^{p}, \mathbf{0}\right) \rightarrow\left(\mathbb{R}^{p}, \mathbf{0}\right)$ such that $f \circ \phi=\psi \circ g$. Therefore, the singular sets of the Gauss maps (i.e., the parabolic sets of $M^{i}$ ) correspond to each other by the condition (1). In general, the $\mathcal{A}$-equivalence of the Gauss maps does not imply the Lagrangian equivalence of the corresponding Lagrangian submanifolds. Moreover, the above theorem asserts that the pictures of the family of the images of the pedal Gauss maps (the wave front propagations) are also corresponding. In the next section we consider the detailed properties of the pedal foliations in the case for surfaces in $\mathbb{R}^{3}$.

\section{Surfaces in Euclidean 3-space}

In this section we consider surfaces in the Euclidean 3-space. Let $\boldsymbol{X}: U \rightarrow \mathbb{R}^{3}$ be an embedding, where $U \subset \mathbb{R}^{2}$ is an open set. In $\S 3$ we introduced the notion of pedal foliations. When $h^{\pi}$ is constant? This the case that codimension of the pedal foliation is zero. We give a classification of surfaces such that $h^{\pi}$ is constant. 
Proposition 7.1 Let $\boldsymbol{X}: U \rightarrow \mathbb{R}^{3}$ be an regular surface. Suppose that $h^{\pi}$ is constant. Then we have the following cases:

(1) $M=\boldsymbol{X}(U)$ is a subset of a plane,

(2) $M=\boldsymbol{X}(U)$ is a subset of a sphere around the origin,

(3) $M=\boldsymbol{X}(U)$ is a subset of a circular cylinder around the origin $\mathrm{CCY}(r)$, where $\mathrm{CCY}(r)$ is given by a rotation of the standard circular cylinder

$$
\operatorname{SCCY}(r)=\left\{\boldsymbol{x}=\left(x_{1}, x_{2}, x_{3}\right) \in \mathbb{R}^{3} \mid x_{1}^{2}+x_{2}^{2}=r^{2}\right\}
$$

around the origin, where $r>0$,

(4) $M=\boldsymbol{X}(U)$ is a subset of a circular cone $\mathrm{CCO}(r, a)$, where $\operatorname{CCO}(r, a)$ is given by a rotation of the standard circular cone

$$
\operatorname{SCCO}(r, a)=\left\{\boldsymbol{x}=\left(x_{1}, x_{2}, x_{3}\right) \in \mathbb{R}^{3} \mid x_{1}^{2}+x_{2}^{2}-r^{2}\left(x_{3}-a\right)^{2}=0, x_{3}>a\right\}
$$

around the origin, where $r>0$,

Proof. Suppose that $\boldsymbol{X}^{T} \equiv \mathbf{0}$. Then $\left\langle\boldsymbol{X}^{\perp}(u), \mathbb{G}(u)\right\rangle=\langle\boldsymbol{X}(u), \mathbb{G}(u)\rangle=h^{\pi}(u)=r$, so that $\boldsymbol{X}^{\perp}(u)=r \mathbb{G}(u)$. Therefore, $\langle\boldsymbol{X}(u), \boldsymbol{X}(u)\rangle=r^{2}$. This means that $M=\boldsymbol{X}(U)$ is a subset of a sphere around the origin with the radius $|r|$.

We consider the case $\boldsymbol{X}^{T} \not \equiv \mathbf{0}$. Then we have two sets $U_{1}=\left\{u \in U \mid \boldsymbol{X}^{T}(u) \neq 0\right\}$ and $U_{2}=\left\{u \in U \mid \boldsymbol{X}^{T}(u)=0\right\}$. It is clear that $U_{1}$ is an open set and $U_{1} \cup U_{2}=U$. By the assumption, $U_{1} \neq \emptyset$. Moreover, by Corollary 3.3, $U_{1} \subset K^{-1}(0)$. If $U_{2}=\emptyset$, then $M$ is a developable surface. Suppose that $U_{2} \neq \emptyset$. If $U_{2} \cap K^{-1}(0)$ has an interior point $v_{0} \in U$, then there exists an open neighbourhood $V$ of $v_{0}$ such that $\boldsymbol{X}^{T} \mid V \equiv \mathbf{0}$. By the previous argument, $\boldsymbol{X}(V)$ is a part of a sphere, so that $K(v) \neq 0$ on $V$. This is a contradiction, so that $U_{2} \cap K^{-1}(0)$ has no interior points. Thus, we have $\partial U_{1}=U_{2} \cap K^{-1}(0)$. Moreover, if $U \backslash K^{-1}(0)$ is non-empty, then $U \backslash K^{-1}(0) \subset U_{2}$. By the above arguments, we have Int $U_{2}=U \backslash K^{-1}(0)$. In this case $\boldsymbol{X}\left(\operatorname{Int} U_{2}\right)$ is a part of a sphere and $\boldsymbol{X}\left(U_{1}\right)$ is a developable surface. Therefore, we may suppose that $U_{2}=\emptyset$, so that $M$ is a developable surface.

It is classically known that developable surfaces is determined completely as follows [21]: A developable surface is classified into one of the following cases:

(1) a part of a cylindrical surface,

(2) a part of a cone,

(3) a part of a tangent surface,

(4) the glue of the above three surfaces.

Suppose that $M$ is a part of a cylindrical surface. It is parametrized at least locally by $\boldsymbol{X}(s, u)=\gamma(s)+u \boldsymbol{e}$, where $\gamma(s)$ is a unit speed space curve and $\boldsymbol{e}$ is a unit constant vector. Moreover, we can choose that $\gamma(s)$ is a planar curve such that $\boldsymbol{t}(s) \perp \boldsymbol{e}$, where $\boldsymbol{t}(s)=\boldsymbol{\gamma}^{\prime}(s)$ is a unit tangent vector. In this case the unit normal of $\boldsymbol{X}(s, u)$ is given by $\boldsymbol{n}(s, u)=\boldsymbol{t}(s) \times \boldsymbol{e}$. Therefore, we have $h^{\pi}(s, u)=\langle\boldsymbol{\gamma}(s)+u \boldsymbol{e}, \boldsymbol{t}(s) \times \boldsymbol{e}\rangle=\langle\boldsymbol{\gamma}(s), \boldsymbol{t}(s) \times \boldsymbol{e}\rangle=\langle\boldsymbol{\gamma}(s) \times \boldsymbol{t}(s), \boldsymbol{e}\rangle=r$. By the Frenet-Serret formulae, we have $0=\left(\partial h^{\pi} / \partial s\right)(s, u)=\langle\gamma(s) \times \kappa(s) \boldsymbol{n}(s)$, e $\rangle$, where $\boldsymbol{n}(s)$ is the principal normal vector. Since $\boldsymbol{\gamma}$ is a planar curve and $\boldsymbol{t}(s) \perp \boldsymbol{e}$, we have $\boldsymbol{\gamma}(s) \times \kappa(s) \boldsymbol{n}(s) \equiv \mathbf{0}$. If $\kappa \equiv 0$ on some interval $I$, then $\boldsymbol{\gamma}$ is a line on $I$, so that $\boldsymbol{X} \mid I \times \mathbb{R}$ is a part of a plane. If $\kappa \neq 0$ on some interval $I, \gamma(s)$ and $\boldsymbol{n}(s)$ are parallel on $I$. There exists $\lambda(s)$ such that $\gamma(s)=\lambda(s) \boldsymbol{n}(s)$, so that $\pm \lambda(s)=\langle\lambda(s) \boldsymbol{e}, \boldsymbol{e}\rangle=\langle\lambda(s) \boldsymbol{n} \times \boldsymbol{t}(s), \boldsymbol{e}\rangle=\langle\gamma(s) \times \boldsymbol{t}(s), \boldsymbol{e}\rangle=r$. It follows that $\boldsymbol{X}(s, u)=r \boldsymbol{n}(s)+u \boldsymbol{e}$. This means that $\boldsymbol{X}(s, u)$ is on a circular cylinder around the origin for $s \in I$. 
Suppose that $M$ is a part of a cone. It is parametrized at least locally by $\boldsymbol{X}(s, u)=\boldsymbol{a}+u \boldsymbol{\delta}(s)$, where $\boldsymbol{\delta}(s)$ is a unit speed spherical curve and $\boldsymbol{a}$ is a constant vector. Then $\boldsymbol{t}(s)=\boldsymbol{\delta}^{\prime}(s)$ is a unit vector such that $\boldsymbol{\delta}(s)$ and $\boldsymbol{t}(s)$ are orthogonal. In this case the unit normal of $\boldsymbol{X}(s, u)$ is given by $\boldsymbol{n}(s, u)=\boldsymbol{\delta}(s) \times \boldsymbol{t}(s)=\boldsymbol{d}(s)$. The moving frame $\{\boldsymbol{\delta}, \boldsymbol{t}, \boldsymbol{d}\}$ is called a Sabban frame along the spherical curve $\boldsymbol{\delta}$ [16]. We have the Frenet-Serret type formulae:

$$
\left\{\begin{array}{l}
\boldsymbol{\delta}^{\prime}(s)=\boldsymbol{t}(s) \\
\boldsymbol{t}^{\prime}(s)=\kappa_{g}(s) \boldsymbol{d}(s)-\boldsymbol{\delta}(s), \\
\boldsymbol{d}^{\prime}(s)=-\kappa_{g}(s) \boldsymbol{t}(s)
\end{array}\right.
$$

where $\kappa_{g}(s)=\left\langle\boldsymbol{t}^{\prime}(s), \boldsymbol{d}(s)\right\rangle$ is the geodesic curvature of $\boldsymbol{\delta}(s)$. By the assumption, we have $h^{\pi}(s, u)=\langle\boldsymbol{a}, \boldsymbol{d}(s)\rangle=r$ and $0=\left(\partial h^{\pi} / \partial s\right)(s, u)=\left\langle\boldsymbol{a}, \boldsymbol{d}^{\prime}(s)\right\rangle=\left\langle\boldsymbol{a},-\kappa_{g}(s) \boldsymbol{t}(s)\right\rangle$. If $\kappa_{g} \equiv 0$, then $\boldsymbol{\delta}$ is a geodesic in the unit sphere, so that it is a great circle. Moreover, $\boldsymbol{d}(s)$ is a constant vector. This means that $\boldsymbol{X}(s, u)$ is in the plane $\langle\boldsymbol{x}, \boldsymbol{d}\rangle=r$. If $\kappa_{g}(s) \neq 0$ on some interval $I$, then $\langle\boldsymbol{a}, \boldsymbol{t}(s)\rangle=0$ on $I$. Therefore $\boldsymbol{t}(s)$ is a planer curve, so that $\boldsymbol{\delta}$ is a curve in the plane $\langle\boldsymbol{x}, \boldsymbol{a}\rangle=c$. If we consider a vector $\widetilde{\boldsymbol{a}}=\left(c /\|\boldsymbol{a}\|^{2}\right) \boldsymbol{a}$, then $\boldsymbol{\delta}(s)-\widetilde{\boldsymbol{a}}$ is orthogonal to $\boldsymbol{a}$. Moreover, we have

$$
\langle\boldsymbol{\delta}(s)-\widetilde{\boldsymbol{a}}, \boldsymbol{\delta}(s)-\widetilde{\boldsymbol{a}}\rangle=\langle\boldsymbol{\delta}(s), \boldsymbol{\delta}(s)\rangle-2\langle\boldsymbol{\delta}(s), \widetilde{\boldsymbol{a}}\rangle+\langle\widetilde{\boldsymbol{a}}, \widetilde{\boldsymbol{a}}\rangle=1-\frac{c^{2}}{\|\boldsymbol{a}\|^{2}} .
$$

This means that $\boldsymbol{\delta}(s)$ is in the circle in the plane $\langle\boldsymbol{x}, \boldsymbol{a}\rangle=c$. We have the case (4).

Finally, we suppose that $M$ is a part of a tangent surface. It is parametrized at least locally by $\boldsymbol{X}(s, u)=\boldsymbol{\gamma}(s)+u \boldsymbol{t}(s)$, where $\boldsymbol{\gamma}(s)$ is a unit speed curve with $\kappa(s) \neq 0$ and $\boldsymbol{t}(s)$ is the unit tangent vector of $\boldsymbol{\gamma}$. We denote the principal normal vector by $\boldsymbol{n}(s)$ and the binormal vector by $\boldsymbol{b}(s)$ of $\boldsymbol{\gamma}$. It is known that the unit normal vector of $\boldsymbol{X}(s, u)$ is the binormal vector $\boldsymbol{b}(s)$ of $\boldsymbol{\gamma}$. Therefore, we have $h^{\pi}(s, u)=\langle\boldsymbol{\gamma}(s)+u \boldsymbol{t}(s), \boldsymbol{b}(s)\rangle=\langle\boldsymbol{\gamma}(s), \boldsymbol{b}(s)\rangle=r$. Thus, we have $\partial h^{\pi} / \partial s(s, u)=\langle\boldsymbol{t}(s), \boldsymbol{b}(s)\rangle+\left\langle\boldsymbol{\gamma}(s), \boldsymbol{t}^{\prime}(s)\right\rangle=-\tau(s)\langle\boldsymbol{\gamma}(s), \boldsymbol{n}(s)\rangle=0$, where $\tau(s)$ is the torsion of $\boldsymbol{\gamma}$. If $\tau \equiv 0$, then $\boldsymbol{\gamma}$ is a planer curve, so that $\boldsymbol{t}(s)$ is also planer. Therefore $\boldsymbol{X}(s, u)$ is part of a plane. If $\tau(s) \neq 0$ on an interval $I$, then $\langle\boldsymbol{\gamma}(s), \boldsymbol{n}(s)\rangle=0$, so that there exist $\lambda(s), \mu(s)$ such that $\gamma(s)=\lambda(s) \boldsymbol{t}(s)+\mu(s) \boldsymbol{b}(s)$. Since $\mu(s)=\langle\boldsymbol{\gamma}(s), \boldsymbol{b}(s)\rangle=r$, we have $\boldsymbol{\gamma}(s)=\lambda(s) \boldsymbol{t}(s)+r \boldsymbol{b}(s)$ for $s \in I$. It follows that

$$
\boldsymbol{t}(s)=\gamma^{\prime}(s)=\lambda^{\prime}(s) \boldsymbol{t}(s)+\lambda(s) \boldsymbol{t}^{\prime}(s)+r \boldsymbol{b}^{\prime}(s)=\lambda^{\prime}(s) \boldsymbol{t}(s)+\kappa(s)(\lambda(s)-r) \boldsymbol{n}(s) .
$$

Therefore, we have $\lambda^{\prime}(s)=1$ and $\lambda(s)=r$. This is a contradiction. If $\kappa(s)=0$ on an interval $J$, then $\gamma(s)$ is a line such that the direction is given by $\boldsymbol{t}(s)$. Then $\boldsymbol{X}(s, u)$ is a line on $J \times \mathbb{R}$, which is singular. This completes the proof.

Since the leaf of the pedal foliation on a surface is a regular curve in generic, we consider generic properties of regular curves on a surface. Let $\gamma: I \rightarrow U \subset \mathbb{R}^{2}$ be a regular curve such that $\overline{\boldsymbol{\gamma}}=\boldsymbol{X} \circ \boldsymbol{\gamma}$ is a unit speed curve. Then $\boldsymbol{t}(s)=\overline{\boldsymbol{\gamma}}^{\prime}(s)$ is the unit tangent vector field. Let $\boldsymbol{n}_{\gamma}(s)$ is the unit normal vector field of $M=\boldsymbol{X}(U)$ along $\overline{\boldsymbol{\gamma}}$. We define the relative normal vector field of $\overline{\boldsymbol{\gamma}}$ by $\boldsymbol{e}(s)=\boldsymbol{n}_{\gamma}(s) \times \boldsymbol{t}(s)$. Then we have the following Frenet-Serret type formulae:

$$
\left\{\begin{array}{l}
\boldsymbol{t}^{\prime}(s)=\kappa_{g}(s) \boldsymbol{e}(s)+\kappa_{n}(s) \boldsymbol{n}_{\gamma}(s), \\
\boldsymbol{e}^{\prime}(s)=-\kappa_{g}(s) \boldsymbol{t}(s)+\tau_{g}(s) \boldsymbol{n}_{\gamma}(s), \\
\boldsymbol{n}_{\gamma}^{\prime}(s)=-\kappa_{n}(s) \boldsymbol{t}(s)-\tau_{g}(s) \boldsymbol{e}(s),
\end{array}\right.
$$

where $\kappa_{n}(s)$ is the normal curvature, $\kappa_{g}(s)$ is the geodesic curvature and $\tau_{g}(s)$ is the geodesic torsion. The frame $\left\{\boldsymbol{t}(s), \boldsymbol{e}(s), \boldsymbol{n}_{\gamma}(s)\right\}$ is called the Darboux frame. It is known that 
1) $\gamma$ is an asymptotic curve of $M$ if and only if $\kappa_{n}=0$,

2) $\gamma$ is a geodesic of $M$ if and only if $\kappa_{g}=0$,

3) $\gamma$ is a principal curve of $M$ if and only if $\tau_{g}=0$.

By the Frenet-Serret type formulae, $\mathbb{G} \circ \boldsymbol{\gamma}=\boldsymbol{n}_{\gamma}$ is singular at $s$ if and only if $\kappa_{n}(s)=\tau_{g}(s)=0$.

Proposition 7.2 Let $\mathcal{L}_{u_{0}}^{\pi}(M)$ be a non-singular pedal leaf through $u_{0} \in U$. Let $\boldsymbol{\gamma}: I \rightarrow U$ be a regular curve such that $\overline{\boldsymbol{\gamma}}=\boldsymbol{X} \circ \boldsymbol{\gamma}$ is a parametrization of the pedal leaf $\mathcal{L}_{u_{0}}^{\pi}(M)$ with $\boldsymbol{\gamma}\left(s_{0}\right)=u_{0}$. Then the following conditions are equivalent:

(1) The pedal Gauss map $\mathbb{G} \mid \mathcal{L}_{u_{0}}^{\pi}(M)$ is singular at $p=\boldsymbol{X}\left(u_{0}\right)$,

(2) $\kappa_{n}\left(s_{0}\right)=\tau_{g}\left(s_{0}\right)=0$,

(3) The tangent line $T_{p} \mathcal{L}_{u_{0}}^{\pi}(M)$ gives the principal direction with the zero principal curvature.

Proof. Since $\mathbb{G} \mid \mathcal{L}_{u_{0}}^{\pi}(M)$ is parametrized by $\mathbb{G} \circ \boldsymbol{\gamma}=\boldsymbol{n}_{\gamma}$, it has been already shown that the conditions (1) and (2) are equivalent. The condition (2) means that $\boldsymbol{t}\left(s_{0}\right)$ directs both the asymptotic and the principal directions. Here, we have $T_{p} \mathcal{L}_{u_{0}}^{\pi}(M)=\left\langle\boldsymbol{t}\left(s_{0}\right)\right\rangle_{\mathbb{R}}$ Therefore, the conditions (2) and (3) are equivalent.

We now revisit the characterizations of the cusp point of the Gauss map in [2]. There are some geometric characterization of the cusp point of the Gauss map. We add extra new conditions to the characterizations of the cusp point of the Gauss map here.

Theorem 7.3 Suppose that the Gauss map $\mathbb{G}$ of $M=\boldsymbol{X}(U)$ is stable. Then the parabolic locus $K^{-1}(0)$ is a regular curve and the following conditions are equivalent:

(1) $p=\boldsymbol{X}\left(u_{0}\right)$ is a cusp of the Gauss map $\mathbb{G}$,

(2) $p=\boldsymbol{X}\left(u_{0}\right)$ is a swallowtail of the cylindrical pedal $\mathbb{C P}_{M}$,

(3) The central pedal leaf $\mathcal{L}_{u_{0}}^{\pi}(M)$ is tangent to the parabolic locus $K^{-1}(0)$. Moreover, for any $\varepsilon>0$, there exist $u_{1} \neq u_{2} \in U$ such that $\left|u_{1}-u_{2}\right|<\varepsilon$, the tangent planes at $u_{1}, u_{2}$ are different but $h^{\pi}\left(u_{1}\right)=h^{\pi}\left(u_{2}\right)$ and the pedal leaf $\mathcal{L}_{u_{1}}^{\pi}(M)=\mathcal{L}_{u_{2}}^{\pi}(M)$ is transverse to the parabolic locus $K^{-1}(0)$,

(4) The family of the images of the pedal Gauss maps $\left\{\mathbb{G} \mid \mathcal{L}_{u}^{\pi}(M)\right\}_{h^{\pi}(u)=t \in\left(\mathbb{R}, t_{0}\right)}$ is the swallowtail bifurcation on $S^{2}$.

Proof. In the previous contexts (cf. [12]), it has been already known that the conditions (1) and (2) are equivalent. Since the cylindrical pedal $\mathbb{C P}_{M}$ is a graphlike wave front and the family of images of Pedal Gauss maps is the family of corresponding small fronts, so the conditions (2) and (4) are equivalent by using the classification of non-degenerate graphlike Legendrian unfoldings in [11, Theorem 2.3]. By Proposition 7.2, the tangent line $T_{p} \mathcal{L}_{u_{0}}^{\pi}(M)$ gives the principal direction with the vanishing principal curvature. There is a characterization of the cusp point of the Gauss map in [3] that the principal direction with the vanishing principal curvature is tangent to the parabolic point at the point and transverse at the other points. This means that the conditions (1) and (3) are equivalent.

\section{References}

[1] V. I. Arnol'd, S. M. Gusein-Zade and A. N. Varchenko, Singularities of Differentiable Maps vol. I. Birkhäuser (1986). 
[2] T. Banchoff, T. Gaffney and C. McCrory, Cusps of Gauss mappings. Research Notes in Mathematics, 55. Pitman (1982).

[3] D. Bleeker and L. Wilson, Stability of Gauss maps. Illinois J. Math. 22, (1978), 279-289

[4] Th. Bröcker, Differentiable Germs and Catastrophes. London Mathematical Society, Lecture Note Series 17, Cambridge University Press, (1975)

[5] J. W. Bruce, The duals of generic hypersurfaces. Math. Scand. 49 (1981), 36-60.

[6] J. W. Bruce and P. J. Giblin, Curves and singularities. A geometrical introduction to singularity theory. Second edition. Cambridge University Press (1992).

[7] J. W. Bruce and M. C. Romero Fuster, Duality and orthogonal projections of curves and surfaces in Euclidean 3-space. Quart. J. Math. Oxford Ser. (2) 42 (1991), 433-441.

[8] A. Dimca, Topics on real and complex singularities. Advanced Lectures in Mathematics (1987).

[9] M. P. Do Carmo, Differential Geometry of Curves and Surfaces. Prentice-Hall (1976).

[10] V. V. Goryunov, Projections of Generic Surfaces with Boundaries. Adv. Soviet Math. 1 (1990), 157-200.

[11] S. Izumiya, Perestroikas of optical wave fronts and graphlike Legendrian unfoldings. J. Differential Geom. 38 (1993), 485-500.

[12] S. Izumiya, Differential Geometry from the viewpoint of Lagrangian or Legendrian singularity theory. in Singularity Theory (ed., D. Chéniot et al), World Scientific (2007), 241-275.

[13] S. Izumiya and M. Takahashi, Spacelike parallels and evolutes in Minkowski pseudo-spheres. Journal of Geometry and Physics. 57 (2007), 1569-1600.

[14] S. Izumiya and M. Takahashi, Caustics and wave front propagations: Applications to differential geometry. Banach Center Publications. Geometry and topology of caustics. 82 (2008) $125-142$.

[15] S. Izumiya and M. Takahashi, On caustics of submanifolds and canal hypersurfaces in Euclidean space. Topology and its Appl. 159 (2012) 501-508.

[16] J. J. Koenderink, Solid shape. MIT Press, Cambridge (1990).

[17] E. E. Landis, Tangential singularities. Funct. Anal. Appli., 15 (1981), 103-114

[18] J. Martinet, Singularities of Smooth Functions and Maps. London Math. Soc. Lecture Note Series, Cambridge Univ. Press, 58 (1982).

[19] O. A. Platonova, Singularities in the problem of the quickest way round an obstrract. Funct. Anal. Appli.,15 (1981), 147-148

[20] M. C. Romero Fuster, Sphere stratifications and the Gauss map. Proceedings of the Royal Soc. Edinburgh, 95A (1983), 115-136

[21] I. Vaisman, A first coourse in differential geometry. A Series of Monographs and Textbooks 80, MARCEL DEKKER, (1984) 
[22] V. M. Zakalyukin, Reconstructions of fronts and caustics depending on a parameter and versality of mappings. J. Soviet Math. 27 (1983), 2713-2735.

[23] V. M. Zakalyukin, Envelope of Families of Wave Fronts and Control Theory. Proc. Steklov Inst. Math. 209 (1995), 114-123.

Shyuichi Izumiya, Department of Mathematics, Hokkaido University, Sapporo 060-0810, Japan. e-mail: izumiya@math.sci.hokudai.ac.jp

Masatomo Takahashi, Muroran Institute of Technology, Muroran 050-8585, Japan.

e-mail: masatomo@mmm.muroran-it.ac.jp 